\section{Acute infection linked to increased risk of vascular events}

A recent study by Smeeth and colleagues has shown that the risk of stroke or myocardial infarction is increased in the days following an acute respiratory or urinary tract infection. Common vaccinations, however, were not associated with an increase in the risk of vascular events.

By use of data from the United Kingdom General Practice Research Database, the authors performed a case-series analysis of all patients who had experienced a myocardial infarction or stroke on a known date within a 5-year period. Data were then extracted on influenza, tetanus and pneumococcus vaccinations as well as acute bronchitis, influenza, undefined chest infections and acute urinary tract infections. No difference was observed in the incidence of myocardial infarction or stroke before and after vaccinations. In patients who had a systemic respiratory infection, however, the risk of both events was significantly increased compared with the period before infection (incidence ratios 1-3 days after diagnosis of the infection were 4.95 and 3.19 for a first myocardial infarction or stroke, respectively). Urinary tract infections had a similar effect on the risk of vascular events, although the increased incidence was less dramatic. Recurrent stroke or myocardial infarction were also more likely following acute infection.

Smeeth et al. conclude that acute infection is likely to have a causal role in prompting cardiovascular events. Since respiratory and urinary tract infections are dissimilar, systemic inflammation is likely to underlie this phenomenon.

Original article Smeeth L et al. (2004) Risk of myocardial infarction and stroke after acute infection or vaccination. N Engl J Med 351: 2611-2618

\section{Alcohol and CHD risk}

The apparent cardioprotective effect of moderate alchohol consumption might be mediated by its anti-inflammatory effects. Imhof et al. have tested this hypothesis by examining the relationship between alcohol consumption and markers of systemic inflammation.

This observational study used MONICA samples from Augsburg, Glasgow and Lille, representing the general populations of Germany, Scotland and France, respectively. These three samples differed substantially in terms of alcohol consumption, socioeconomic factors and cardiovascular disease risk. The 7,887 participants provided information about their intake of beer, wine and spirits. In all three samples, those whose alcohol intake was moderate (up to $40 \mathrm{~g}$ per day) had lower plasma concentrations of C-reactive protein and fibrinogen, lower plasma viscosity and lower white blood cell counts than those who drank heavily or not at all. This effect persisted after adjustment for lifestyle factors and other potential confounders and was independent of the type of alcohol consumed, suggesting that a moderate intake was associated with a reduction in systemic inflammation.

Since interventional studies of alcohol consumption are not appropriate for ethical reasons, the authors propose that longterm, prospective, observational studies should be carried out to further examine the relationship between alcohol and systemic inflammation.

Original article Imhof A et al. (2004) Overall alcohol intake, beer, wine, and systemic markers of inflammation in western Europe: results from three MONICA samples (Augsburg, Glasgow, Lille). Eur Heart J 25: 2092-2100

\section{GLOSSARY}

MONICA

Multinational Monitoring Of

Trends And Determinants

In Cardiovascular Disease Surveys 